\title{
A High-Order Solution for the Distribution of Target Registration Error in Rigid-Body Point-Based Registration
}

\author{
Mehdi Hedjazi Moghari ${ }^{1}$ and Purang Abolmaesumi ${ }^{1,2}$ \\ ${ }^{1}$ Department of Electrical and Computer Engineering, Queen's University, Canada \\ ${ }^{2}$ School of Computing, Queen's University, Canada \\ purang@cs.queensu.ca
}

\begin{abstract}
Rigid registration of pre-operative surgical plans to intraoperative coordinates of a patient is an important step in computerassisted orthopaedic surgery. A good measure for registration accuracy is the target registration error (TRE) which is the distance after registration between a pair of corresponding points not used in the registration process. However, TRE is not a deterministic value, since there is always error in the localized features (points) utilized in the registration. In this situation, the distribution of TRE carries more information than TRE by itself. Previously, the distribution of TRE has been estimated with the accuracy of the first-order approximation. In this paper, we analytically approximate the TRE distribution up to at least the second-order accuracy based on the Unscented Kalman Filter algorithm.
\end{abstract}

\section{Introduction}

The problem of finding the best translation vector and rotation matrix that accurately registers matching point pairs in two data sets, corrupted by isotropic noise, is also called the absolute orientation problem. This problem has been shown to have closed-form solutions [1]. However, reported solutions will end up with imperfect registration when there is error in extracting the feature points employed for registering the two data sets (these extracted points might also be called fiducial points 2, and therefore, the error in extracting them is named fiducial localization error, FLE). Hence, some measures are required to estimate the level of the registration accuracy. To analyze the accuracy of point-based registration algorithms, Maurer et al., has proposed a measure called the target registration error (TRE) $[3]$ which is the distance between the corresponding point pairs not used in the registration process. The properties of TRE have been under investigation for a long period of time. Fitzpatrick et al., in 1998, analytically derived an approximation of the root mean squared value of TRE 2[4]. Later on, in 2001, they mathematically explored an approximation of the TRE distribution [5]. However, the work only presents the first-order approximation of TRE and it does not give an accurate result when the structure of data sets are not equivalent (the moments of data sets about each axis are substantially 
different) [5. In what follows, we derive an estimation of the distribution of TRE along with its mean squared value, with the accuracy of at least second-order Taylor series approximation. Furthermore, a closed-form solution for computing the distribution of TRE is obtained and is verified by means of numerical simulations. Furthermore, it is shown that the proposed algorithm outperforms the algorithm addressed in [5] most importantly when data sets have different moments about each axis.

\section{Method}

Let's assume that the points in one space (moving data set) is shown by $\mathbf{U}_{3 \times N}$ as a matrix whose columns correspond to the position vectors of points in that space. $\mathbf{Y}_{3 \times N}$ represents the corresponding points in the second space (fixed data set). The $i$ th columns of $\mathbf{U}$ and $\mathbf{Y}$ represent a pair of corresponding points in the two spaces. As in [5], we also, make a simplifying assumption that FLE in the moving space $\mathbf{U}$ is negligible (identically zero) in comparison to FLE in the fixed data set $\mathbf{Y}$, by adding the variance of point localization error in the moving data set, $\sigma_{U}^{2}$, to the one in the fixed data set, $\sigma_{Y}^{2}$. The goal is to determine a rotation matrix $\mathbf{R}$ and a translation vector $\mathbf{t}$ from the following observation model:

$$
\mathbf{y}_{1: i}=\mathbf{R}_{\left(\theta_{\mathbf{x}}, \theta_{\mathbf{y}}, \theta_{\mathbf{z}}\right)} \mathbf{u}_{1: i}+\mathbf{t}_{\left(t_{x}, t_{y}, t_{z}\right)}+\mathbf{n}_{1: i},
$$

where $\mathbf{y}_{1: i}=\left[\mathbf{y}_{1}^{T}, \ldots, \mathbf{y}_{i}^{T}\right]^{T}, \mathbf{u}_{1: i}=\left[\mathbf{u}_{1}^{T}, \ldots, \mathbf{u}_{i}^{T}\right]^{T}, \mathbf{n}_{1: i}=\left[\mathbf{n}_{1}^{T}, \ldots, \mathbf{n}_{i}^{T}\right]^{T}$ and $i=$ $1, \ldots, N . \mathbf{t}=\left[t_{x}, t_{y}, t_{z}\right]^{T}$ is the translation vector along orthogonal coordinate frame's axes and $\theta_{x}, \theta_{y}$ and $\theta_{z}$ are the rotational angles about the orthogonal coordinate frame's axes, respectively. $\mathbf{n}_{i}$ is fiducial localization error (FLE) for point $i$ which is assumed to be a zero-mean Gaussian random vector with covariance matrix $\boldsymbol{\Sigma}_{n_{i}}=\left(\sigma_{U}^{2}+\sigma_{Y}^{2}\right) I$, where $I$ is the identity matrix. In other words, $\mathbf{n}_{i}$ is the error occurring in collecting features (points) from a rigid object for the registration, and it is assumed to be isotropic, and to have the same distribution at each point. In this work, we have used the Euler angles to represent the rotation matrix R. We have accounted for this representation's singularity points in our simulations by limiting the range in which each rotation angle could change. However, the same analysis presented here is valid for other rotation matrix representations such as the Quaternions and the rotation around a helical axis. Let us define the state vector $\mathbf{x}$ as, $\mathbf{x}=\left[t_{x}, t_{y}, t_{z}, \theta_{x}, \theta_{y}, \theta_{z}\right]^{T}=\left[\mathbf{x}_{t}^{T}, \mathbf{x}_{\theta}^{T}\right]^{T} . \mathbf{x}_{R}$ and $\mathbf{x}_{t}$ is defined to be $\left[\theta_{x}, \theta_{y}, \theta_{z}\right]^{T}$ and $\left[t_{x}, t_{y}, t_{z}\right]^{T}$, respectively. Since Equation (1) is a nonlinear function of $\mathbf{x}$, we utilize the Unscented Kalman Filter (UKF) algorithm [6] to estimate the optimum state vector $\mathbf{x}$ in the sense of minimum mean square error. UKF is an algorithm for sequentially updating the state vector $\mathbf{x}$ (pose parameters) which is governed by the nonlinear system, Equation (11). Sequentially estimating the pose parameters while pairs of corresponding points are being collected, makes real time registration process more feasible. An alternative to this approach is the employment of the Extended Kalman Filter algorithm for estimating the state parameters and their variances, as proposed 
by Pennec et al [7]. Although EKF works for smoothly nonlinear functions, we propose the UKF algorithm since: 1) UKF can be applied to non-differentiable functions, 2) UKF avoids the derivation of Jacobian matrices, and 3) UKF is valid for higher-order (at least second-order) estimation of the Taylor series expansion than the standard EKF algorithm with the same amount of computational complexity. However, 8 shows that UKF and EKF algorithms have equivalent performance for estimating pose parameters; but our investigations [9] illustrate that the UKF algorithm outperforms EKF in two ways. Firstly, UKF requires fewer input points to converge to the correct solution; secondly, it estimates the variance of pose parameters more accurately than EKF.

\subsection{The UKF-Based Registration Algorithm}

Let us assume that the state model is defined as $\mathbf{x}_{i}=\mathbf{x}_{i-1}+\mathcal{N}\left(0, \boldsymbol{\Sigma}_{Q}\right)$ with the initial value and covariance matrix $\mathbf{x}_{0}$ and $\mathbf{P}_{x}^{0}$, respectively. $\mathcal{N}\left(0, \boldsymbol{\Sigma}_{Q}\right)$ is a zero-mean Gaussian random vector with a covariance matrix $\boldsymbol{\Sigma}_{Q}$. It is assumed that the state vector $\mathbf{x}$ has the Gaussian distribution. By these assumptions, one can estimate the state vector $\mathbf{x}$ from the observation model as follows:

1) Predict the state vector and its covariance matrix from the state model as: $\hat{\mathbf{x}}_{i}^{-}=\hat{\mathbf{x}}_{i-1}$, and $\mathbf{P}_{\hat{\mathbf{x}}_{i}^{-}}=\mathbf{P}_{\hat{\mathbf{x}}_{i-1}}+\boldsymbol{\Sigma}_{Q}$.

2) Append the $i$ th point from the moving data set $\mathbf{U}$ to the already collected points from that data set, and estimate their corresponding points' positions in the fixed data set, $\mathbf{Y}$, by using the predicted state vector in step one as $\hat{\mathbf{y}}_{1: i}=\mathbf{R}_{\left(\hat{\theta}_{\mathbf{x}}, \hat{\theta}_{\mathbf{y}}, \hat{\theta}_{\mathbf{z}}\right)} \mathbf{u}_{1: i}+\mathbf{t}_{\left(\hat{\mathbf{t}}_{\mathbf{x}}, \hat{\mathbf{t}}_{\mathbf{y}}, \hat{\mathbf{t}}_{\mathbf{z}}\right)}$.

3) Compute the error in the estimated corresponding points in the data set $\mathbf{Y}$, $\left(\mathbf{y}_{1: i}-\hat{\mathbf{y}}_{1: i}\right)$, to update the state vector and its covariance matrix as follows:

$$
\hat{\mathbf{x}}^{i}=\hat{\mathbf{x}}_{i}^{-}+\mathbf{K}_{i}\left(\mathbf{y}_{1: i}-\hat{\mathbf{y}}_{1: i}\right), \quad \mathbf{P}_{\hat{\mathbf{x}}_{i}}=\mathbf{P}_{\hat{\mathbf{x}}_{i}^{-}}-\mathbf{K}_{i} E\left[\mathbf{y}_{1: i} \mathbf{y}_{1: i}^{T}\right] \mathbf{K}_{i}^{T},
$$

where $\mathbf{K}_{i}=E\left[\mathbf{x}_{i} \mathbf{y}_{1: i}^{T}\right] / E\left[\mathbf{y}_{1: i} \mathbf{y}_{1: i}^{T}\right]$. Since the observation model, Equation (1), is nonlinear, the Unscented Transform (UT) [10] is used to compute $E\left[\mathbf{x}_{i} \mathbf{y}_{1: i}^{T}\right]$ and $E\left[\mathbf{y}_{1: i} \mathbf{y}_{1: i}^{T}\right]$, respectively. This algorithm is iterated through all the points in the moving data set $\mathbf{U}$ to estimate the optimal state vector.

\subsection{Verification of the UKF-Based Registration Algorithm}

We examine the UKF-based registration algorithm by registering two random data sets each containing 30 points for 6,000 trials. The moving data set $\mathbf{U}$ is drawn uniformly from a cube within the rang of $\pm 250 \mathrm{~mm}$. The fixed data set $\mathbf{Y}$ is generated by applying a random transformation on the moving data set. The random transformation is generated by uniformly drawing the rotational and translational parameters along all axes within the range of $\pm 80^{\circ}$ and $\pm 80 \mathrm{~mm}$, respectively. Also, zero-mean Gaussian random noise is added to the fixed data set to simulate FLE which is assumed to be isotropic. The distribution of FLE is considered to be $\mathcal{N}\left(0,1 \mathrm{~mm}^{2}\right)$ and to be the same at each point in the fixed data 
set $\mathbf{Y}$. The UKF-based registration algorithm is then employed to register these two data sets. It is assumed that if $\left\|\mathbf{x}_{t}-\hat{\mathbf{x}}_{t}\right\|^{2}$ and $\left\|\mathbf{x}_{R}-\hat{\mathbf{x}}_{R}\right\|^{2}$ are less than $2 \mathrm{~mm}^{2}$ and 2 degree ${ }^{2}$, respectively, the algorithm is converged. In this simulation the UKF-based registration algorithm converges for $\% 97$ of the time. Figure 1 shows the error distribution of estimated translational and rotational parameters. Mean and variance of error of the estimated transformation parameters are listed in Table 1. This simulation is repeated with different distributions of FLE, as $\mathcal{N}\left(0,5 \mathrm{~mm}^{2}\right)$ and $\mathcal{N}\left(0,10 \mathrm{~mm}^{2}\right)$, respectively. In these experiments, the algorithm converges $\% 92$ and $\% 77$ of the time, respectively. By increasing the variance of FLE in the fixed data set, the number of registration points should be raised to attain the same accuracy and convergence rate.

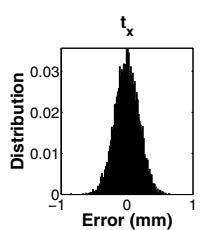

$\theta_{\mathbf{x}}$
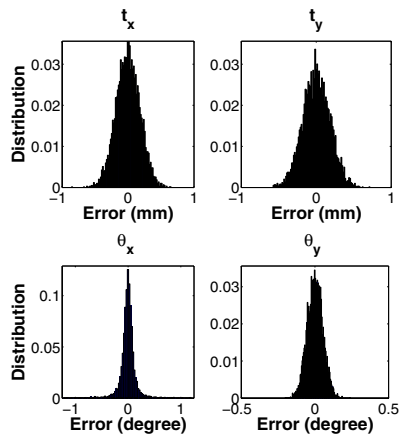

$\theta_{y}$
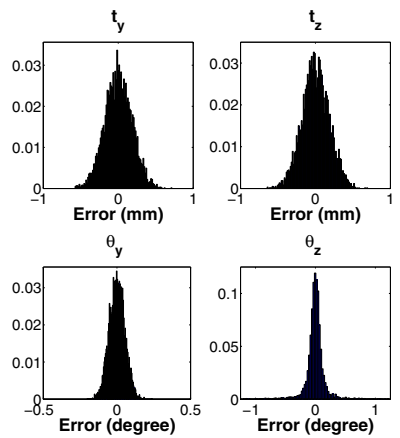

$\theta_{\mathbf{z}}$

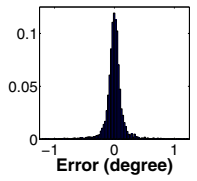

Fig. 1. Distribution of the estimated transformation parameters using the UKF-based registration algorithm over 5824 trials
Table 1. Mean and variance of error of estimated translational $(\mathrm{mm})$ and rotational (degree) parameters

\begin{tabular}{c|l|l}
\hline \multirow{2}{*}{$\begin{array}{c}\text { Estimation } \\
\text { Error }\end{array}$} & \multicolumn{2}{|l}{ Experiment I } \\
\cline { 2 - 3 } & Mean & Var. \\
\hline$\hat{t}_{x}-t_{x}$ & -0.002 & 0.033 \\
$\hat{t}_{y}-t_{y}$ & 0.002 & 0.033 \\
$\hat{t}_{z}-t_{z}$ & 0.001 & 0.033 \\
$\hat{\theta}_{x}-\theta_{x}$ & -0.004 & 0.031 \\
$\hat{\theta}_{y}-\theta_{y}$ & 0.007 & 0.003 \\
$\hat{\theta}_{z}-\theta_{z}$ & -0.003 & 0.031 \\
\hline
\end{tabular}

\section{Derivation of the TRE Distribution}

Let us assume that the registration is performed between the fixed and the moving data sets and the transformation parameters are converged on the true solution. Let $\mathbf{r}$ be a point, other than the ones used in the registration algorithm, where we would like to compute the target registration error, $\mathrm{TRE}_{r}$. The registration error at the target point $\mathbf{r}$ can be determined as:

$$
\mathbf{e}(\mathbf{r})=\hat{\mathbf{R}} \mathbf{r}+\hat{\mathbf{t}}-\mathbf{R r}-\mathbf{t}=(\hat{\mathbf{R}}-\mathbf{R}) \mathbf{r}+(\hat{\mathbf{t}}-\mathbf{t})=\Delta \mathbf{R r}+\Delta \mathbf{t},
$$

where $\Delta \mathbf{R}=\hat{\mathbf{R}}-\mathbf{R}$ and $\Delta \mathbf{t}=\hat{\mathbf{t}}-\mathbf{t}$. Assuming that the registration algorithm has converged on the true values of transformation parameters $(\hat{\mathbf{R}}$ and $\hat{\mathbf{t}}$ are unbiased, and $E[\Delta \mathbf{R}]$ and $E[\Delta \mathbf{t}]$ are zero), based on the defined state model, $\Delta \mathbf{t}$ is a zero-mean Gaussian distributed random vector with covariance matrix $\boldsymbol{\Sigma}_{\Delta t}=E\left[\Delta \mathbf{t} \Delta \mathbf{t}^{T}\right]$, where $\boldsymbol{\Sigma}_{\Delta t}$ can be easily derived from Equation (2) as follows:

$$
\mathbf{P}_{\hat{\mathbf{x}}}=E\left[(\mathbf{x}-\hat{\mathbf{x}})(\mathbf{x}-\hat{\mathbf{x}})^{T}\right]=\left(\begin{array}{cc}
\boldsymbol{\Sigma}_{\Delta t} & \boldsymbol{\Sigma}_{\Delta \theta t} \\
\boldsymbol{\Sigma}_{\Delta \theta t} & \boldsymbol{\Sigma}_{\Delta \theta}
\end{array}\right)_{6 \times 6},
$$


Assuming that the registration algorithm has converged on the true solution, $(\Delta \mathbf{R r})$, in Equation (3), is a zero-mean random vector with an unknown distribution. Its mean $(E[\Delta \mathbf{R r}]=0)$ and covariance matrix $\left(\boldsymbol{\Sigma}_{\Delta R r}=E\left[(\Delta \mathbf{R r})(\Delta \mathbf{R r})^{T}\right]\right)$ can be also estimated using the Unscented Transform [10], since $(\Delta \mathbf{R r})$ is a nonlinear function of the Gaussian random vector $\mathbf{x}_{\theta} \cdot \mathbf{x}_{\theta}$ has mean $\hat{\mathbf{x}}_{\theta}$ and covariance matrix $\boldsymbol{\Sigma}_{\theta}$ that can be determined from Equations (2) and (4), respectively. Therefore, the distribution of error vector e can be approximated by a zero-mean Gaussian distribution with covariance matrix $E\left[\mathbf{e e}^{T}\right], \mathcal{N}\left(0, E\left[\mathbf{e e}^{T}\right]\right)$, where $E\left[\mathbf{e e}^{T}\right]$ can be determined as follows:

$$
E\left[\mathbf{e e}^{T}\right]=E\left[\Delta \mathbf{R r}(\Delta \mathbf{R r})^{T}\right]+2 E\left[(\Delta \mathbf{R r}) \Delta \mathbf{t}^{T}\right]+E\left[\Delta \mathbf{t} \Delta \mathbf{t}^{T}\right]
$$

$\boldsymbol{\Sigma}_{(\Delta R r \Delta t)}=E\left[(\Delta \mathbf{R r}) \Delta \mathbf{t}^{T}\right]$ is the cross-correlation matrix between $(\Delta \mathbf{R r})$ and $\Delta \mathbf{t}$ that can be computed by using the Unscented Transform and propagating the estimated state vector $\hat{\mathbf{x}}$ and its covariance matrix $\mathbf{P}_{\hat{\mathbf{x}}}$ through the nonlinearity $\left([\Delta \mathbf{R r}] \Delta \mathbf{t}^{T}\right)$. Equation (5) can be simplified as, $E\left[\mathbf{e e}^{T}\right]=\boldsymbol{\Sigma}_{\Delta R r}+$ $2 \boldsymbol{\Sigma}_{(\Delta R r \Delta t)}+\boldsymbol{\Sigma}_{\Delta t}$. By defining the error vector $\mathbf{e}$ as $\left[e_{x}, e_{y}, e_{z}\right]^{T}$, we have $T R E_{r}=$ $\sqrt{e_{x}^{2}+e_{y}^{2}+e_{z}^{2}}$, where $e_{x}, e_{y}$ and $e_{z}$ are zero-mean Gaussian random variables. Since $\mathrm{TRE}_{r}$ is a distance error and distance is invariant to rotations, error vector e can be arbitrary rotated in a space without varying the distribution of $\mathrm{TRE}_{r}$. Hence, one may rotate e such that its covariance matrix, $E\left[\mathbf{e e}^{T}\right]$, becomes diagonal and therefore its components become independent zero-mean Gaussian random variables. Lets assume that $\boldsymbol{\Sigma}_{e}$ is the diagonalized covariance matrix of $E\left[\mathbf{e e}^{T}\right]=\operatorname{diag}\left[\lambda_{1}, \lambda_{2}, \lambda_{3}\right]$, where $\lambda_{1}, \lambda_{2}$ and $\lambda_{3}$ are the variances of the first, the second and the third elements of the rotated error vector e, respectively. We first determine the distribution of the $\mathrm{TRE}_{r}^{2}$ from which the distribution of $\mathrm{TRE}_{r}$ can be easily derived. $\mathrm{TRE}_{r}^{2}$ is the summation of three squared independent zero-mean Gaussian random variables, $e_{1}, e_{2}$ and $e_{3}$, with the variances of $\lambda_{1}, \lambda_{2}$ and $\lambda_{3}$, respectively. It means that the distribution of $\mathrm{TRE}_{r}^{2}$ is the convolution of each squared Gaussian random variable as $p d f_{\left(T R E_{r}^{2}\right)}(x)=$ $p d f_{\left(e_{1}^{2}\right)}(x) * p d f_{\left(e_{2}^{2}\right)}(x) * p d f_{\left(e_{3}^{2}\right)}(x)$, where $p d f_{\left(e_{i}^{2}\right)}(x)=\frac{1}{\sqrt{2 \pi x \lambda_{i}}} \exp \left(-\frac{x}{2 \lambda_{i}}\right), x \geq 0$. By performing the convolutions, the distribution of $\mathrm{TRE}_{r}^{2}$ can be obtained as:

$$
p d f_{\left(T R E_{r}^{2}\right)}(x)=\frac{\exp \left(-\frac{x}{2 \lambda_{3}}\right)}{2 \sqrt{2 \pi \lambda_{1} \lambda_{2} \lambda_{3}}} \int_{0}^{x} \frac{\exp \left(\frac{-\lambda}{4}\left[\frac{1}{\lambda_{1}}+\frac{1}{\lambda_{2}}-\frac{2}{\lambda_{3}}\right]\right)}{\sqrt{(x-\lambda)}} I_{0}\left(\frac{\lambda}{4}\left[\frac{1}{\lambda_{1}}-\frac{1}{\lambda_{2}}\right]\right) d \lambda,(6)
$$

where $I_{0}(x)=\frac{1}{\pi} \int_{0}^{\pi} \exp (x \cos \phi) d \phi$. From Equation (6), the distribution of TRE $\mathrm{T}_{r}$ is derived as follows:

$$
p d f_{\left(T R E_{(r)}\right)}(x)=\frac{x \exp \left(-\frac{x^{2}}{2 \lambda_{3}}\right)}{\sqrt{2 \pi \lambda_{1} \lambda_{2} \lambda_{3}}} \int_{0}^{x^{2} \exp \left(\frac{-\lambda}{4}\left[\frac{1}{\lambda_{1}}+\frac{1}{\lambda_{2}}-\frac{2}{\lambda_{3}}\right]\right)} \underset{\sqrt{\left(x^{2}-\lambda\right)}}{ } I_{0}\left(\frac{\lambda}{4}\left[\frac{1}{\lambda_{1}}-\frac{1}{\lambda_{2}}\right]\right) d \lambda .
$$




\section{Locus of the Points with the Same Mean Square TRE}

In this section, we explain how to estimate mean squared value of $\mathrm{TRE}_{r}$, $E\left[T R E_{r}^{2}\right]$, using the variances estimated from the UKF-based registration algorithm. By using Equation (3), mean square $\mathrm{TRE}_{r}$ can be formulated as:

$$
\begin{aligned}
E\left[T R E_{r}^{2}\right] & =E\left[\mathbf{e}^{T} \mathbf{e}\right]=E\left[(\Delta \mathbf{R r}+\Delta \mathbf{t})^{T}(\Delta \mathbf{R r}+\Delta \mathbf{t})\right], \\
& =\mathbf{r}^{T} E\left[\Delta \mathbf{R}^{T} \Delta \mathbf{R}\right] \mathbf{r}+2 E\left[\Delta \mathbf{t}^{T} \Delta \mathbf{R}\right] \mathbf{r}+E\left[\Delta \mathbf{t}^{T} \Delta \mathbf{t}\right],
\end{aligned}
$$

where $\boldsymbol{\Sigma}_{\Delta R}=E\left[\Delta \mathbf{R}^{T} \Delta \mathbf{R}\right]$ is the covariance matrix of the random matrix $\Delta \mathbf{R}$ and is a symmetric positive definite matrix, and $\mathbf{v}=E\left[\Delta \mathbf{t}^{T} \Delta \mathbf{R}\right]^{T}$. By defining $\Delta \mathbf{t}=\left[\Delta t_{x}, \Delta t_{y}, \Delta t_{z}\right]^{T}$, Equation (8) can be rewritten as:

$$
E\left[T R E_{r}^{2}\right]=\mathbf{r}^{T} \boldsymbol{\Sigma}_{\Delta R} \mathbf{r}+2 \mathbf{v}^{T} \mathbf{r}+E\left[\Delta t_{x}^{2}\right]+E\left[\Delta t_{y}^{2}\right]+E\left[\Delta t_{z}^{2}\right],
$$

where $E\left[\Delta t_{x}^{2}\right]=\Sigma_{\Delta t}^{11}, E\left[\Delta t_{y}^{2}\right]=\Sigma_{\Delta t}^{22}$ and $E\left[\Delta t_{z}^{2}\right]=\Sigma_{\Delta t}^{33}$ can be obtained from Equation (4) by considering that $\Sigma_{t}^{i j}$ is the $i j$ th element of the covariance matrix $\boldsymbol{\Sigma}_{\Delta t}$. Also, since $\Delta \mathbf{R}$ is a nonlinear function of the Gaussian random vector $\mathbf{x}_{\theta}$ with mean of $\hat{\mathbf{x}}_{\theta}$ and covariance matrix of $\boldsymbol{\Sigma}_{\theta}$, covariance matrix $\boldsymbol{\Sigma}_{\Delta R}$ can be estimated using UT. Vector $\mathbf{v}$ is computed by propagating $\hat{\mathbf{x}}$ and $\mathbf{P}_{\hat{\mathbf{x}}}$ through the nonlinearity $\left(\Delta \mathbf{t}^{T} \Delta \mathbf{R}\right)$ and using UT. Therefore, by estimating the variance of each translational components, covariance matrix $\boldsymbol{\Sigma}_{\Delta R}$ and $\mathbf{v}, E\left[T R E_{r}^{2}\right]$ can be easily obtained from Equation (9). To find the loci of the points with the same mean squared values of TRE, let's represent the target point $\mathbf{r}$ as a vector $\left[r_{x}, r_{y}, r_{z}\right]^{T}$. Then, Equation (9) can be expanded as follows:

$$
\begin{aligned}
E\left[T R E_{r}^{2}\right] & =\Sigma_{\Delta R}^{11} r_{x}^{2}+\Sigma_{\Delta R}^{22} r_{y}^{2}+\Sigma_{\Delta R}^{33} r_{z}^{2}+2 \Sigma_{\Delta R}^{12} r_{x} r_{y}+2 \Sigma_{\Delta R}^{13} r_{x} r_{z}+2 \Sigma_{\Delta R}^{23} r_{y} r_{z} \\
& +2 v_{1} r_{x}+2 v_{2} r_{y}+2 v_{3} r_{z}+E\left[\Delta t_{x}^{2}\right]+E\left[\Delta t_{y}^{2}\right]+E\left[\Delta t_{z}^{2}\right],
\end{aligned}
$$

where $\Sigma_{\Delta R}^{i j}$ is assumed to be the $i j$ th element of $\boldsymbol{\Sigma}_{\Delta R}$ and $v_{i}$ is the $i$ th element of vector $\mathbf{v}$. The locus of the points that have the same mean square TRE can be derived from Equation (10). For example, the locus of the points with the same $E\left[T R E^{2}\right]$ on a plane parallel to $\mathbf{x}$ and $\mathbf{y}$ axes is either an ellipse or a circle depending on the covariance matrix $\boldsymbol{\Sigma}_{\Delta R}$.

\section{Results}

In order to verify our derivations, several simulations are performed. In the first simulation, we choose $N$, the number of points in data sets to be 30 . We generate the moving data set $\mathbf{U}$ by drawing $N$ points uniformly within a cube in the range of $\pm 100 \mathrm{~mm}$. Also, one target position is selected randomly from a cube in the range of $\pm 200 \mathrm{~mm}$. In order to generate the fixed data set $\mathbf{Y}$, we perturb independently $x, y$, and $z$ components of each point in $\mathbf{U}$ by zero-mean Gaussian random noise with variance of $6 \mathrm{~mm}^{2}$. In this way we produce the same model as that used by Fitzpatrick [5]. The moving data set $\mathbf{U}$ is registered to 
the fixed one $\mathbf{Y}$ and the TRE is measured at the target point. The distribution of TRE is generated by repetitions of perturbation and registration steps for 100,000 times. The generated distribution is then used as the gold standard. We employ the UKF-based registration algorithm to register these two data sets to compute the variance of the estimated transformation parameters. Equation (7) is then used to estimate the distribution of TRE at the target position. We have repeated this simulation with different target positions for 500 times and have compared the estimated distribution of TRE with the gold standard one (generated from simulations) by determining the distance among the two distributions as [1], $d\left(f_{1}, f_{2}\right)=\int_{-\infty}^{\infty} f_{1}(\lambda) \log \frac{2 f_{1}(\lambda)}{f_{1}(\lambda)+f_{2}(\lambda)} d(\lambda)+\int_{-\infty}^{\infty} f_{2}(\lambda) \log \frac{2 f_{2}(\lambda)}{f_{1}(\lambda)+f_{2}(\lambda)} d(\lambda)$, where $f_{1}$ and $f_{2}$ are two different distributions. Means and variances of the distance among the computed distributions over 500 trials are listed in Table 2 , Additionally, in each trial, $E\left[T R E^{2}\right]$ is estimated. The normalized mean and the normalized variance of error in the estimation of $E\left[T R E^{2}\right]$ are listed in Table 3 . Tables 2 and 3 show that the estimated distribution of TRE and the estimated $E\left[T R E^{2}\right]$ using the proposed and Fitzpatrick's methods are almost the same as the ones generated by numerical simulations. This has been predictable, since for uniformly distributed data sets (which have the same moments along all axis), the first-order approximation is sufficient to estimate the distribution of TRE. In the second simulation it is shown that when data sets have significantly different moments along each axis, the first-order approximation may not be sufficient to accurately estimate the TRE distribution. In this simulation, we generate data sets with different variances along $\mathbf{x}, \mathbf{y}$ and $\mathbf{z}$ directions. Basically, $x, y$, and $z$ coordinates of points in the moving data set are generated randomly from uniform distributions in the range of $\pm 10 \mathrm{~mm}, \pm 30 \mathrm{~mm}$, and $\pm 60 \mathrm{~mm}$, respectively. We have repeated the same simulation procedure as we performed in the first simulation. Figure 2 shows the estimated distributions of TRE and the one obtained by numerical simulations at a randomly selected target point $\mathbf{r}=[-6.35,-33.2,-167.13]^{T}$. As before, distance between the estimated distribution of TRE and the simulated one is computed and shown in Table 2, Also, the normalized mean and the normalized variance of error in the estimation of $E\left[T R E^{2}\right]$ are listed in Table [3. It can be concluded from Tables 2, 3 and Figure 2 that when data sets are not uniformly distributed along all axes, the first-order approximation is not accurate enough to estimate the distribution of TRE, whereas our proposed higher order approximation closely follows the ground truth simulation results in this case. In the final simulation, the locus of the target points in the same plane parallel to $\mathbf{x}$ and $\mathbf{y}$ axes, which have the same $E\left[T R E^{2}\right]$, is obtained. To do so, we have randomly generated 30 points within a cube in the range of $\pm 100 \mathrm{~mm}$ as the moving data set $\mathbf{U}$. Also, a target position $\mathbf{p}_{1}=[59.9569,-45.2688,-29.6124]^{T}$ is chosen randomly within a cube in the range of $\pm 200 \mathrm{~mm}$. Zero-mean Gaussian random noise with the variance of $6 \mathrm{~mm}^{2}$ in each direction is added to each point in the moving data set to generate the fixed data set $\mathbf{Y}$. The UKF-based registration algorithm is used to register these data sets. Using Equation (10), locus of the points which have the same $E\left[T R E^{2}\right]$ as $\mathbf{p}_{1}$ in a plane parallel to $\mathbf{x y}$ plane is drawn in Figure 3 . 
Table 2. Mean and variance of distances between the distributions, estimated by the Fitzpatrick's and the UKF algorithms and the simulated one (gold standard)

\begin{tabular}{l|l|l|l|l}
\hline Simulation & \multicolumn{2}{|c|}{ UKF } & \multicolumn{2}{c}{ Fitzparick's } \\
\cline { 2 - 5 } Results & Mean & Var. & Mean & Var. \\
\hline \hline Sim.-I & 0.0006 & $1 \times 10^{-7}$ & 0.0008 & $3 \times 10^{-7}$ \\
\hline Sim.-II & 0.073 & $2 \times 10^{-4}$ & 0.235 & $9 \times 10^{-4}$ \\
\hline
\end{tabular}

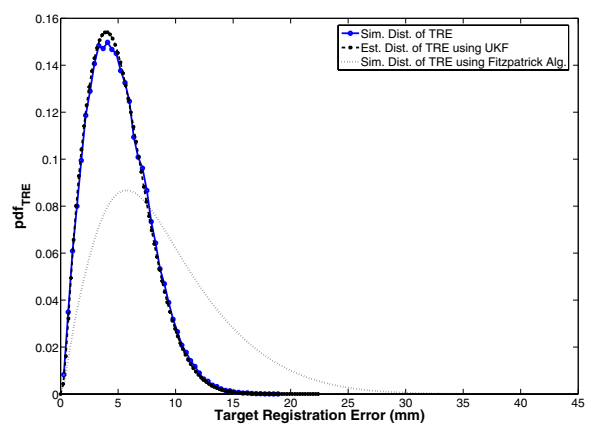

Fig. 2. Estimated TRE distributions using Fitzpatrick's and UKF-based registration algorithms
Table 3. Normalized mean and normalized variance of error in the estimation of mean square TRE using the Fitzpatrick's and the UKF algorithms

\begin{tabular}{l|l|l|l|r}
\hline $\begin{array}{c}\text { Simulation } \\
\text { Results }\end{array}$ & \multicolumn{2}{|c|}{ UKF } & \multicolumn{2}{c}{ Fitzparick's } \\
\cline { 2 - 5 } & Mean & Var. & Mean & Var. \\
\hline \hline Sim.-I & $\% 1.9$ & $\% 0.008$ & $\% 1.5$ & $\% 0.19$ \\
\hline Sim.-II & $\% 10.68$ & $\% 0.023$ & $\% 42.79$ & $\% 0.19$ \\
\hline
\end{tabular}

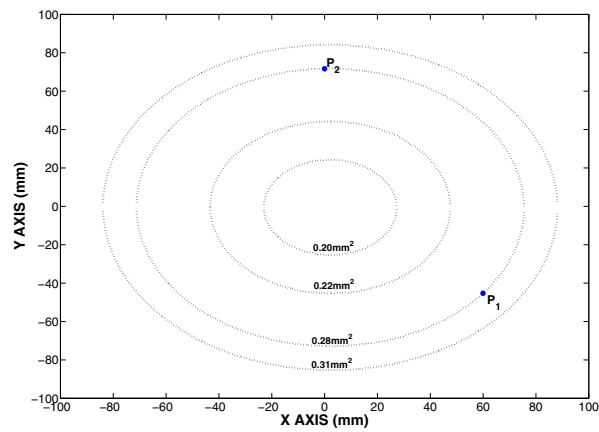

Fig. 3. Loci of the points with the same $E\left[T R E^{2}\right]$ values

\section{Discussion}

We have proposed a new method to estimate the distribution of target registration error (TRE) at an arbitrary target position with the accuracy of at least second-order Taylor series expansion. It is shown that when data sets have different moments along each axis, the first-order approximation may not be accurate enough to estimate the TRE distribution; however, the proposed algorithm still accurately calculates the distribution of TRE. To derive the TRE distribution, the UKF-based registration algorithm is presented. The algorithm is able to estimate the registration transformation parameters along with their variances. Using the estimated variances, new closed-form formulas for computing the distribution of TRE and $E\left[T R E^{2}\right]$ (Equations (7) and (9)) are drawn, respectively.

\section{References}

1. Lorusso, A., Eggert, D.W., Fisher, R.B.: A comparison of four algorithms for estimating 3-D rigid transformations. In: British Mach. Vis. Conf. (1995)

2. Fitzpatrick, J.M., West, J.B., Maurer, C.R.: Predicting error in rigid-body pointbased registration. IEEE Trans. Med. Imag. 17(5) (1998) 694-702 
3. Maurer, C.R., Fitzpatrick, J.M., Wang, M.Y., Galloway, R.L., Macinnas, R.J., s. Allen, G.: Registration of head volume images using implantable fiducial markers. Tech. Rep. CS-96-03, Dept. Comp. Sci., Vanderbilt Univ., Nashvill, TN (1996)

4. Fitzpatrick, J.M., West, J.B., Maurer, C.R.: Derivation of expected registration error for point-based rigid-body registration. In: SPIE- MedI. Volume 3338. (1998) $16-27$

5. Fitzpatrick, J.M., West, J.B.: The distribution of target registration error in rigidbody point-based registration. IEEE Trans. on Med. Imag. 20(9) (2001) 917-927

6. Julier, S.J., Uhlmann, J.K.: Unscented filtering and nonlinear estimation. In: Proc. of the IEEE (Invited paper). Volume 92. (2004) 401-422

7. Pennec, X., Thrion, J.P.: A frame work for uncertaity and validation of 3D registration methods based on points and frames. Comp. Vis. 25(3) (1997) 203-229

8. LaViola, Jr., J.J.: A comparison of unscented and extended kalman filtering for estimating quaternion motion. In: Proc. American Cont. Conf. (2003) 2435-2440

9. Moghari, M.H., Abolmaesumi, P.: Comparing the unscented and extended kalman filter algorithms in the rigid-body point-based registration. In: EMBC2006. (2006)

10. Julier, and J. K. Uhlmann, S.J.: The Scaled Unscented Transformation. In: Proc. IEEE American Cont. Conf., Anchorage AK, USA, IEEE (2002) 4555-4559

11. Lin, J.: Divergence measures based on the shannon entropy. IEEE Trans. Inf. Theory 37(1) (1991) 145-251 